\title{
Risk factors for acute ischaemic stroke in young adults in South India
}

\author{
K Lipska, P N Sylaja, P S Sarma, K R Thankappan, V R Kutty, R S Vasan, K Radhakrishnan
}

J Neurol Neurosurg Psychiatry 2007;78:959-963. doi: 10.1136/jnnp.2006.106831

See end of article for authors' affiliations

......................

Correspondence to:

Dr K Radhakrishnan, Department of Neurology,

Sree Chitra Tirunal Institute for Medical Sciences and Technology, Trivandrum695011 , Kerala, India;

krk@sctimst.ac.in

Received 13 September 2006 Revised 30 November 2006 Accepted 1 January 2007

Published Online First

12 January 2007

Background: Stroke is a leading cause of death and disability in developing countries, afflicting individuals at a young age. The contribution of established vascular risk factors to ischaemic stroke in young adults has not been evaluated systematically in Indians.

Methods: We conducted a case control study in 214 South Indian patients with first acute ischaemic stroke that occurred between the ages of 15 and 45 years, 99 age and sex matched hospital controls and 96 community controls. We compared the prevalence of the following risk factors: smoking, elevated blood pressure, high fasting blood glucose and abnormal lipids.

Results: Compared with community controls, stroke patients had a higher prevalence of smoking (multivariable adjusted odds ratio (OR) $7.77,95 \% \mathrm{Cl} 1.93$ to 31.27 ), higher systolic blood pressure (OR per SD increment of 1.88, 95\% Cl 1.01 to 3.49) and fasting blood glucose (OR per SD increment of 4.55, $95 \% \mathrm{Cl} 1.63$ to 12.67), but lower high density lipoprotein (HDL) cholesterol (OR per SD increment of 0.17, $95 \% \mathrm{Cl} 0.09$ to 0.30 ). Compared with hospital controls, stroke patients had a higher prevalence of smoking IOR 3.95, 95\% Cl 1.61 to 9.71) and lower HDL cholesterol (OR per SD increment $0.27,95 \% \mathrm{Cl} 0.17$ to 0.44 ). The presence of $\geqslant 3$ metabolic syndrome components was associated strongly with stroke (OR 4.76, $95 \% \mathrm{Cl} 1.93$ to 11.76 ; OR $2.09,95 \% \mathrm{Cl} 1.06$ to 4.13 ) compared with community and hospital controls. Conclusions: Key components of the metabolic syndrome and smoking are associated with ischaemic stroke in young South Indian adults. Our observations underscore the importance of targeting adolescents and young adults for screening and prevention to reduce the burden of ischaemic stroke in young adults.

S troke is the leading cause of adult disability and is the second commonest cause of death worldwide. ${ }^{1}$ More than two-thirds of the global burden of stroke is borne by developing countries, where the average age of patients with stroke is 15 years younger that in developed countries. ${ }^{2}$ The available limited data indicate that stroke occurring in young people is more often atherothrombotic in origin in developing countries, ${ }^{3-5}$ in contrast with developed countries where arterial dissection and cardioembolic aetiologies predominate..$^{6-9}$

We hypothesised that ischaemic stroke occurring in young Indians may be a manifestation of accelerated cerebrovascular atherosclerosis, paralleling the early age of onset of cardiovascular diseases noted for this population. ${ }^{10}$ Additionally, based on prior data on increased propensity for insulin resistance among South Asians, ${ }^{11}{ }^{12}$ we postulated that ischaemic stroke in young adults in India will be associated with the combination of risk factors that identify the metabolic syndrome. We tested our hypotheses by conducting a case control study of adults between the ages of 15 and 45 years belonging to the South Indian state of Kerala with a first ischaemic stroke.

\section{SUBJECTS AND METHODS \\ Study site}

This study was conducted at the Sree Chitra Tirunal Institute for Medical Sciences and Technology (SCTIMST), Trivandrum, the capital city of the South Indian state of Kerala, situated on the south west coast of the Indian peninsula. This tertiary referral centre for cardiological, neurological and vascular disorders receives patients from all over Kerala as well as the neighbouring state of Tamil Nadu. All participants provided informed consent, and the study protocol was approved by the institutional review board of SCTIMST.

\section{Study subjects \\ Cases}

We recruited 214 consecutive patients, aged 15-45 years, hospitalised to SCTIMST with a first ischaemic stroke during the period 1995-2001. We used the WHO definition of stroke, ${ }^{13}$ and the diagnosis of ischaemic stroke was supported in every patient by axial CT and/or MRI. Patients were excluded for the following reasons: prior history of stroke; presentation to the hospital $>6$ months after stroke onset; haemorrhagic stroke; and intracranial sinus-venous thromboses.

\section{Controls}

We recruited two sets of controls: 99 hospital controls and 96 community controls. The hospital controls were gathered prospectively during the period January to June 2002 by two investigators (KL, PNS) from eligible individuals, aged 1545 years, who presented to SCTIMST without a prior history of stroke or heart disease. They included patients (and their family members) attending the general neurology or epilepsy clinics for follow-up of neurological disorders other than stroke (or transient ischaemic attack) or complications of vascular disease, and patients admitted to general neurology inpatient wards for neurological disorders other than stroke or transient ischaemic attack, or complications of vascular disease, and who were not currently receiving steroid treatment. Hospital controls were matched to cases for age (within 5 years) and sex. As community controls, we recruited 96 subjects from a community based sample of individuals aged 20-45 years residing in the Trivandrum city, who were recruited in 1999

Abbreviations: HDL, high density lipoprotein; SCTIMST, Sree Chitra Tirunal Institute for Medical Sciences and Technology; TOAST, Trial of Org 10172 in Acute Stroke Treatment 
Table 1 Characteristics of stroke cases and controls

\begin{tabular}{|c|c|c|c|c|c|}
\hline & $\begin{array}{l}\text { Community } \\
\text { controls } \\
(\mathrm{n}=96)\end{array}$ & p Value & $\begin{array}{l}\text { Cases } \\
(n=214)\end{array}$ & p Value & $\begin{array}{l}\text { Hospital } \\
\text { controls } \\
(n=99)\end{array}$ \\
\hline Age $(y)^{*}$ & $33(8)$ & 0.04 & $35(7)$ & 0.03 & $33(8)$ \\
\hline Men (\%) & 42 & $<0.001$ & 66 & 0.52 & 63 \\
\hline Current smoking (\%) & 6 & $<0.001$ & 37 & $<0.001$ & 12 \\
\hline \multicolumn{6}{|l|}{ Blood pressure } \\
\hline Systolic blood pressure $(\mathrm{mm} \mathrm{Hg})$ & $122(12)$ & 0.008 & $127(17)$ & 0.44 & $125(14)$ \\
\hline Diastolic blood pressure (mm Hg) & $81(7)$ & 0.25 & $82(11)$ & 0.14 & $80(9)$ \\
\hline Hypertension (\%) & 17 & 0.001 & 36 & 0.09 & 25 \\
\hline Hypertension treatment (\%) & 5 & 0.10 & 12 & 0.10 & 5 \\
\hline \multicolumn{6}{|l|}{ Lipids } \\
\hline Serum total cholesterol $(\mathrm{mmol} / \mathrm{l})$ & $5.55(1.14)$ & 0.41 & $5.38(1.89)$ & 0.79 & $5.32(1.21)$ \\
\hline HDL cholesterol (mmol/l) & $1.40(0.33)$ & $<0.001$ & $0.94(0.30)$ & $<0.001$ & $1.24(0.32)$ \\
\hline Non-HDL cholesterol (mmol/l) & $4.15(1.22)$ & 0.36 & $4.29(1.20)$ & .22 & $4.11(1.08)$ \\
\hline Total cholesterol/HDL ratio & $4.2(1.5)$ & $<0.001$ & $5.9(1.9)$ & $<0.001$ & $4.5(1.2)$ \\
\hline Hypercholesterolaemia (\%) & 33 & 0.50 & 29 & 0.41 & 24 \\
\hline Treatment for dyslipidaemia & Nil & 1.000 & 1 & 0.04 & 5 \\
\hline Triglycerides (mmol/l) & $1.23(0.76)$ & $<0.001$ & $1.61(0.94)$ & 0.19 & $1.47(0.68)$ \\
\hline \multicolumn{6}{|l|}{ Glycaemia } \\
\hline Fasting blood sugar (mmol/l) & $4.34(1.03)$ & $<0.001$ & $5.26(2.53)$ & 0.52 & $5.09(1.83)$ \\
\hline Diabetes mellitus & 4 & 0.02 & 14 & 0.13 & 7 \\
\hline Treatment for diabetes (\%) & 1 & 0.01 & 8 & 0.36 & 5 \\
\hline \multicolumn{6}{|l|}{ Metabolic syndrome traits (\%) } \\
\hline $\begin{array}{l}\mathrm{BP} \geqslant 130 / 85 \mathrm{~mm} \mathrm{Hg} \text { or on antihypertensive treatment } \\
\mathrm{HDL}<40 \text { (men) }<50 \mathrm{mg} / \mathrm{dl} \text { (women) or lipid lowering }\end{array}$ & 26 & $\begin{array}{l}<0.001 \\
<0.001\end{array}$ & 50 & $\begin{array}{c}0.81 \\
<0.001\end{array}$ & 49 \\
\hline treatment & 26 & & 65 & & 35 \\
\hline Triglycerides $\geqslant 150 \mathrm{mg} / \mathrm{dl}$ & 18 & 0.02 & 31 & 0.35 & 25 \\
\hline Fasting blood sugar $\geqslant 100 \mathrm{mg} / \mathrm{dl}$ or on treatment & 4 & $<0.001$ & 23 & 1.000 & 23 \\
\hline$\geqslant 3$ ATP III criteria & 6 & $<0.001$ & 27 & 0.008 & 13 \\
\hline
\end{tabular}

BP, blood pressure; HDL, high density lipoprotein.

Values are mean (SD), unless indicated otherwise.

Smoking was defined as current use of inhaled tobacco.

Hypercholesterolaemia was defined as (1) previously diagnosed by a physician or (2) receiving lipid lowering treatment or (3) total cholesterol $\geqslant 240$ or low density lipoprotein $\geqslant 160$.

Diabetes mellitus was defined as (1) previously diagnosed by a physician (prescribed treatment) or (2) on oral hypoglycaemic agents or insulin or (3) fasting blood sugar $>126$, single measurement available only.

Hypertension was defined as systolic blood pressure $\geqslant 140$ or diastolic blood pressure $\geqslant 90$ or any treatment for high blood pressure.

ATP III criteria: triglycerides $\geqslant 150$, high density lipoprotein $<40$ for men or $<50$ for men, blood pressure $\geqslant 130 / 85$ or on treatment, fasting blood sugar $\geqslant 100$ or on treatment.

$p$ values are based on $t$ test for comparison of means, and Fisher's exact test for proportions.

${ }^{*}$ Age distribution of cases, $\mathrm{n}(\%)$ : $15-20$ years $13(6) ; 21-30$ years 49 (23); $31-40$ years 104 (49); $41-45$ years 48 (22). Age distribution of hospital controls, $\mathrm{n}(\%)$ : $15-20$ years 9 (9); $21-30$ years: 28 (28); $31-40$ years 42 (43); $41-45$ years 20 (20). Age distribution of community controls, $n(\%): 15-20$ years $3(3) ; 21-30$ years 36 (37); $31-40$ years $37(39) ; 41-45$ years 20 (21).

by one of the authors (VR) for a separate investigation of serum lipids and other risk factors for ischaemic heart disease. ${ }^{14}$

\section{Data collection}

For cases, using a structured proforma, the investigators ( $\mathrm{KL}$, PNS) abstracted demographic, clinical and investigative data, and stroke risk factors from the medical records. We ascertained blood pressure, fasting lipids and fasting blood glucose at approximately a week or later after stroke onset, as elevations in blood pressure, hyperglycaemia and lower blood lipids are well documented during the acute phase of stroke. ${ }^{15-17}$ While hospital controls were personally interviewed to gather the required data, the same were accessed from the data already available for community controls. ${ }^{14}$ Blood glucose, total and high density lipoprotein (HDL) cholesterol, and triglycerides were estimated on automated systems with standardised kits using enzymatic methods for both cases and controls (glucose oxidase, cholesterol oxidase, precipitation method and glycerol phosphate oxidase, respectively). Non-HDL cholesterol was estimated by subtracting HDL cholesterol from total cholesterol.

\section{Categorisation of stroke subtypes}

Stroke subtypes were assigned independently by two investigators (KL, PNS; kappa 0.82) according to the TOAST (Trial of
Org 10172 in Acute Stroke Treatment) criteria. ${ }^{18}$ All disagreements between the two investigators were resolved through discussion with the senior neurologist (KR).

\section{Statistical analyses}

We assessed statistical significance of the risk factors between cases and either of the controls (table 1). The variables for multiple logistic regression modelling were selected based on the significance in bivariate analyses and low intercorrelations among them. Two sets of analyses were conducted: one evaluating all ischaemic strokes and another excluding patients with cardioembolism and stroke due to other determined aetiology. Unconditional logistic regression adjusting for matching variables was used because perfect matching was not possible for hospital controls, and community controls were not matched. We adjusted all models for age and sex, and evaluated the following vascular risk factors: systolic and diastolic blood pressure, smoking, blood glucose, serum total cholesterol, HDL cholesterol, non-HDL cholesterol and triglycerides. Other than sex and smoking, all variables were modelled as continuous variables. In additional analyses, we evaluated risk factors as categorical variables using standardised definitions of hypertension (systolic blood pressure $\geqslant 140$ or diastolic blood pressure $\geqslant 90 \mathrm{~mm} \mathrm{Hg}$ or treatment), ${ }^{19}$ hypercholesterolaemia $(\geqslant 6.22 \mathrm{mmol} / \mathrm{l} \quad(240 \mathrm{mg} / \mathrm{dl}))^{20}$ and diabetes 
Table 2 Risk factors associated with ischaemic stroke: results of multivariable logistic regression

\begin{tabular}{|c|c|c|c|c|}
\hline & \multicolumn{2}{|c|}{ Compared with community controls } & \multicolumn{2}{|c|}{ Compared with hospital controls } \\
\hline & Odds ratio $(95 \% \mathrm{Cl})$ & p Value & Odds ratio $(95 \% \mathrm{Cl})$ & p Value \\
\hline \multicolumn{5}{|c|}{$\begin{array}{l}\text { All ischaemic strokes } \\
\text { (A) Models with individual risk factors }\end{array}$} \\
\hline Fasting blood sugar & $4.55(1.63,12.67)$ & 0.004 & $1.17(0.68,2.02)$ & 0.57 \\
\hline Total cholesterol & $0.86(0.44,1.68)$ & 0.66 & $1.92(0.97,3.82)$ & 0.06 \\
\hline HDL cholesterol & $0.17(0.09,0.30)$ & $<0.001$ & $0.27(0.17,0.44)$ & $<0.001$ \\
\hline Triglycerides & $0.70(0.37,1.32)$ & 0.27 & $0.67(0.40,1.11)$ & 0.12 \\
\hline Smoking & $7.77(1.93,31.27)$ & 0.004 & $3.95(1.61,9.71)$ & 0.003 \\
\hline Systolic blood pressure & $1.88(1.01,3.49)$ & 0.045 & $1.13(0.74,1.73)$ & 0.57 \\
\hline \multicolumn{5}{|c|}{ (B) Models with clustering of metabolic syndrome components } \\
\hline$\geqslant 3$ components & $4.76(1.93,11.76)$ & $<0.001$ & $2.09(1.06,4.13)$ & 0.03 \\
\hline \multicolumn{5}{|c|}{$\begin{array}{l}\text { Strokes excluding cardioembolic and strokes due to other aetiologies } \\
\text { (A) Models with individual risk factors }\end{array}$} \\
\hline Fasting blood sugar & $4.33(1.45,12.96)$ & 0.009 & $1.12(0.58,2.19)$ & 0.73 \\
\hline Total cholesterol & $1.10(0.51,2.39)$ & 0.80 & $2.45(1.09,5.51)$ & 0.03 \\
\hline HDL cholesterol & $0.15(0.07,0.30)$ & $<0.001$ & $0.26(0.15,0.46)$ & $<0.001$ \\
\hline Triglycerides & $0.77(0.39,1.50)$ & 0.44 & $0.77(0.42,1.42)$ & 0.41 \\
\hline Smoking & $8.73(2.14,35.68)$ & 0.003 & $5.38(2.01,14.39)$ & 0.001 \\
\hline Systolic blood pressure & $2.35(1.11,4.96)$ & 0.03 & $1.29(0.79,2.12)$ & 0.31 \\
\hline \multicolumn{5}{|c|}{ (B) Models with clustering of metabolic syndrome components } \\
\hline$\geqslant 3$ components & $5.75(2.24,14.76)$ & $<0.001$ & $2.38(1.16,4.87)$ & 0.02 \\
\hline \multicolumn{5}{|c|}{$\begin{array}{l}\text { HDL, high density lipoprotein. } \\
\text { All models adjusted for age and sex. Model A also adjusts for antihypertensive treatment, lipid or blood sugar lowering } \\
\text { treatment. } \\
\text { OR here should be per } 1 \text { SD increment in fasting blood sugar, systolic blood pressure, triglycerides, total and HDL } \\
\text { cholesterol (1 SD for cases; see table 1). }\end{array}$} \\
\hline
\end{tabular}

mellitus ( $\geqslant 7.0 \mathrm{mmol} / \mathrm{l}(126 \mathrm{mg} / \mathrm{dl})) .{ }^{21}$ Effect modification by age was evaluated by modelling appropriate interaction terms for each risk factor (eg, age $\times$ blood pressure). Odds ratios (OR) and their 95\% confidence intervals (CI) were estimated for each risk factor. As data on waist circumference were not available in the medical records, we considered the remaining components of the metabolic syndrome based on revised criteria of the International Diabetes Federation ${ }^{22}$ : raised triglycerides $\geqslant 1.7 \mathrm{mmol} / \mathrm{l}(150 \mathrm{mg} / \mathrm{dl})$ or specific treatment for this lipid abnormality; reduced HDL cholesterol $<1.03 \mathrm{mmol} / \mathrm{l}(40 \mathrm{mg} / \mathrm{dl})$ in men and $<1.29 \mathrm{mmol} / \mathrm{l}(50 \mathrm{mg} / \mathrm{dl})$ in women or specific treatment for this lipid abnormality; raised blood pressure, systolic $\geqslant 130$ or diastolic $\geqslant 85 \mathrm{~mm} \mathrm{Hg}$ or treatment of previously diagnosed hypertension; raised fasting blood glucose $\geqslant 5.6 \mathrm{mmol} / \mathrm{l}(100 \mathrm{mg} / \mathrm{dl})$ or previously diagnosed type 2 diabetes.

\section{RESULTS}

\section{Stroke subtypes}

Based on the TOAST criteria, ${ }^{18}$ there were 54 (25.2\%) patients with cardioembolic stroke (45 major and 9 minor sources of cardiac embolism), 27 (12.6\%) with large artery atherosclerosis and $16(7.5 \%)$ with lacunar infarcts. Twenty-four (11.2\%) strokes were adjudicated as of other determined aetiology: 15 patients with arterial dissection $(7.0 \% ; 8$ carotid system and 7 vertebrobasilar system dissections), 3 possibly related to a haematological/immunoinflammatory condition ( 1 each with lupus erythematosus, primary antiphospholipid antibody syndrome and protein S deficiency), 2 patients with ectasia (one in the vertebrobasilar system, one involving the left middle cerebral artery) and 4 due to other causes ( 1 case of each: Moya Moya disease, Takayasu's arteritis, fibromuscular dysplasia and nephrotic syndrome). Ninety-three strokes (43.5\%) were deemed to be of "indeterminate origin"; of the latter, 23 patients were incompletely investigated (lack of echocardiography and carotid Doppler ultrasonography or angiography), 5 had more than one potential cause for stroke and in 65 patients no cause of stroke was determined despite complete investigation.

\section{Prevalence of vascular risk factors among cases and controls}

The characteristics of the ischaemic stroke cases and the two sets of controls are compared in table 1. Approximately $70 \%$ of cases and hospital controls were aged 20-40 years, compared with $76 \%$ of community controls. Compared with community controls, cases were more likely to be smokers, had higher systolic blood pressure, fasting blood sugar and lower HDL cholesterol levels, and a greater prevalence of hypertension and diabetes mellitus. Compared with hospital controls, cases were more likely to report smoking and had lower HDL cholesterol levels. Mean serum total cholesterol levels in cases did not differ from that in community or hospital controls. A higher proportion of cases had three or more components of the metabolic syndrome compared with both sets of controls.

\section{Multivariable analyses}

In multivariable logistic regression analyses comparing cases to community controls (table 2), smoking (about 8-fold odds compared with non-smokers), higher fasting blood glucose (4.6-fold odds per SD increment) and systolic blood pressure (1.9-fold odds per SD increment) were all associated with stroke. HDL cholesterol was related inversely to stroke $(83 \%$ lower odds per SD increment). Triglyceride levels (table 2) and non-HDL cholesterol (adjusted OR per SD increment 0.90, 95\% CI 0.65 to $1.25, \mathrm{p}=0.55$ ) were not associated with stroke risk. In multivariable models incorporating the ratio of total to HDL cholesterol (instead of the two variables separately), a unit increase in the ratio was associated with a doubling of stroke risk (adjusted OR 2.15, 95\% CI 1.55 to $2.96, \mathrm{p}<0.0001$ ). These relations were consistent when analyses were repeated excluding individuals with cardioembolic stroke and stroke due to other aetiologies (table 2). In analyses assessing effect modification by age, none of the interaction terms for different risk factors was statistically significant. The presence of three or more components of the metabolic syndrome was associated with an approximately 5 -fold stroke risk (table 2 ). In analyses of risk factors as categorical variables, smoking (adjusted OR 7.14, 95\% CI 2.73 to $18.64, \mathrm{p}<0.001$ ) and hypertension 
(adjusted OR 2.46, 95\% CI 1.25 to 4.84 ) were associated with increased stroke risk, whereas a borderline significant association with diabetes was observed (adjusted OR 2.94, 95\% CI 0.93 to $9.30, \mathrm{p}=0.07$ ).

In analyses comparing cases with hospital controls (table 2), smoking was positively related to stroke (near 4-fold odds compared with non-smokers), whereas HDL cholesterol was inversely related (73\% lower odds per SD increment). A unit increase in the ratio of total to HDL cholesterol was associated with a more than 2-fold stroke risk (adjusted OR 2.36, 95\% CI 1.68 to 3.32). High systolic blood pressure, blood sugar, triglyceride levels (table 2 ) and non-HDL cholesterol (adjusted OR per SD increment $1.06,95 \%$ CI 0.77 to $1.47, \mathrm{p}=0.71$ ) were not associated with stroke risk. We did not observe any effect modification by age (all interaction terms were non-significant). The presence of three or more components of the metabolic syndrome was associated with an approximately 2fold stroke risk (table 2). These findings were consistent when analyses were restricted to individuals without cardioembolic stroke or stroke due to other aetiologies (table 2). In analyses of risk factors as categorical variables, only smoking was associated with increased stroke risk (adjusted OR 5.22, 95\% CI 2.49 to $10.94, \mathrm{p}<0.001)$.

\section{DISCUSSION}

To our knowledge, the present investigation is the first case control study that has evaluated risk factors for ischaemic stroke in young adults in India. Two previous case control studies, one in the Parsi community of Bombay (present Mumbai), Central India, ${ }^{23}$ and the other in the South Indian population of Madras (present Chennai), ${ }^{24}$ included completed ischaemic strokes in all age groups.

The selection of controls is usually the most difficult aspect of the case control study design. Although several strategies for control selection are possible, all have certain limitations. ${ }^{25}$ The use of more than one set of controls can test concordance of results across control groups, which increases the validity of the results. ${ }^{25}$ This prompted us to use both community and hospital based controls to investigate the contribution of established vascular risk factors to the development of stroke risk in young adults. The two sets of our controls yielded mostly consistent findings (both in the overall sample and in analyses excluding individuals with cardioembolic and strokes of other aetiology): smoking, low HDL cholesterol levels and the presence of three or more components of the metabolic syndrome emerged as key risk factors for stroke. A stronger association of hyperglycaemia and higher systolic blood pressure with stroke risk was observed in analyses comparing cases with community controls. One potential explanation may be that hospital controls had higher blood sugar and blood pressure levels relative to community controls, in part related to the stress of the hospital environment, diminishing the ability of these factors to distinguish them from cases. Other investigators have reported that hospital controls may resemble cases more, and differ from community controls. ${ }^{26} 27$

Although observational studies from Western countries have emphasised the preponderance of cardiogenic embolism, arterial dissection, procoagulant states and non-atherosclerotic vasculopathies as possible aetiologies, ${ }^{6-9}$ careful analytic comparisons have shown the importance of traditional risk factors in the pathogenesis of stroke in the young adults. ${ }^{28}{ }^{29}$ In the Baltimore-Washington Cooperative Young Stroke Study, ${ }^{28}$ which compared 296 cases of incident ischaemic stroke among black and white adults aged 18-44 years with 1220 community based adults of the same age group, hypertension, diabetes mellitus and current smoking emerged as important risk factors. Similarly, in a comparison of 201 consecutive patients with first onset stroke due to cerebral infarction aged 1555 years and the same number of matched neighbourhood control subjects conducted as part of the Melbourne Risk Factor Study, ${ }^{29}$ hypertension, diabetes mellitus, current smoking, heart disease and long term heavy alcohol consumption were major risk factors. The two case control studies from India that included ischaemic stroke in all age groups suggested that hypertension, diabetes mellitus and smoking are important risk factors for stroke in India as they are worldwide. ${ }^{23}{ }^{24}$ Our observation of the association of the traditional risk factors with stroke in the young adults emphasises their role in the pathogenesis of ischaemic stroke in this age group.

The role of dyslipoproteinaemia in the pathogenesis of cerebrovascular disease in less certain than for coronary artery disease; more consistent association has been noted with low HDL cholesterol and high total cholesterol to HDL cholesterol ratio than with total cholesterol, low density lipoprotein cholesterol and triglycerides. ${ }^{30}{ }^{31}$ Low HDL cholesterol was the only serum lipid index associated with an increased risk of ischaemic stroke among 94 consecutive patients under 45 years admitted to a tertiary care facility in Toulouse, France, when compared with 111 controls of the same age. ${ }^{32}$ In a case control study involving 204 patients with acute ischaemic stroke of all ages from Madras, South India, the authors found that while low HDL cholesterol and high total cholesterol to HDL cholesterol ratio were more frequent among patients, total serum cholesterol, triglycerides and low density lipoprotein cholesterol levels did not significantly differ. ${ }^{24}$ Our results among stroke in young adults are consistent with these observations. HDL cholesterol plays a fundamental role in the regulation of atherogenesis via its effects on reverse cholesterol transport ${ }^{33}$ and vascular remodelling. ${ }^{34}$

Even though fasting blood glucose, systolic blood pressure and lipid profile were not consistently different between cases and the two control groups, the strong association of the conjoint presence of multiple components of the metabolic syndrome with stroke in young adults is a striking observation of the present report. Although several prior reports have associated the metabolic syndrome with stroke risk in middle aged to older adults, ${ }^{35-37}$ to our knowledge, such an association has not been established for stroke in young adults. Overall, our observations add to the growing body of evidence implicating insulin resistance, a precursor of the metabolic syndrome, in the development of vascular disease in young people of South Asian origin. ${ }^{11}{ }^{12}$ Metabolic syndrome in the South Indian population has recently been reported to be associated with Thr54 allele carriers of the Ala54Thr variant of FABP2 gene. ${ }^{38}$ The relative contribution of genetic predisposition and extraneous influences such as food, tobacco and meteorological factors in the pathogenesis of metabolic syndrome among the South Indian population is at present unknown.

The use of standardised criteria for ischaemic stroke, two sets of controls, controls of similar ethnicity and from a limited geographic region strengthen the present investigation. However, we acknowledge the following limitations of our approach. The study was conducted in a tertiary care referral centre for neurological and cardiovascular diseases and may therefore be biased towards more disabling and complicated disease processes. Strokes of indeterminate origin were overrepresented in the sample, presumably because of incomplete investigation of our cases. The modest sample size for controls might have diminished our statistical power to detect more moderate associations with selective risk factors. Additional limitations include the retrospective abstraction of data from medical records, use of single occasion measurements of risk factors, limited ability to characterise a dose-response for key variables such as smoking and lack of waist circumference measurement. 
Despite these limitations, our study incriminates known vascular risk factors in the pathogenesis of ischaemic stroke in young adults, raising the possibility that accelerated atherosclerosis may be the underlying substrate, rather than rarer aetiologies commonly sought in such individuals. Tobacco smoking $^{39}$ and diabetes ${ }^{40}$ are growing problems in the developing countries. Our findings suggest that smoking cessation, identification and treatment of elevated blood pressure, dyslipidaemia and diabetes are critical measures for preventing ischaemic stroke in young adults seen in this geographical region.

\section{ACKNOWLEDGEMENTS}

Dr Lipska was supported by Paul Dudley White and Andrew Sellard Traveling Fellowship of the Harvard Medical School, Boston, Massachusetts, USA, and Dr Vasan received a research grant from the National Heart, Lung and Blood Institute, National Institutes of Health, Bethesda, Maryland, USA.

\section{Authors' affiliations}

K Lipska, Department of Medicine, Brigham and Women's Hospital, Boston University School of Medicine, Boston, Massachusetts, USA P N Sylaja, K Radhakrishnan, Department of Neurology, Sree Chitra Tirunal Institute for Medical Sciences and Technology, Trivandrum, Kerala, India

P S Sarma, K R Thankappan, The Achutha Menon Centre For Health Science Studies, Sree Chitra Tirunal Institute for Medical Sciences and Technology, Trivandrum, Kerala, India

V R Kutty, Health Action by People, Kerala, India

R S Vasan, Cardiology Section and Preventive Medicine and

Epidemiology, Department of Medicine, Boston University School of Medicine, Boston, Massachusetts, USA

\section{Competing interests: None.}

Individual author's contribution to this research and responsibilities: Drs Lipska, Sylaja, Radhakrishnan and Vasan were involved in study conception and design, analysis and interpretation of the data, including writing and critical revision of the report. Dr Sarma provided statistical expertise and directed the analysis and interpretation of the data, and participated in the writing and critical revision of the report. Dr Kutty participated in the collection of data on community controls, the analysis and interpretation of data, and the writing and critical revision of the report. Dr Thankappan contributed to the interpretation of the data, including writing and critical revision of the report. The corresponding author, Dr Radhakrishnan, had full access to all the data in the study, and had final responsibility for the decision to submit for publication.

\section{REFERENCES}

1 Bonita R, Mendis S, Truelsen T, et al. The global stroke initiative. Lancet Neurol 2004;3:391-3.

2 Truelsen T, Bonita R, Jamrozik K. Surveillance of stroke: a global perspective. Int J Epidemiol 2001;30:S1 1-16.

3 Nayak SD, Nair M, Radhakrishnan K, et al. Ischaemic stroke in the young adult: clinical features, risk factors and outcome. Natl Med J India 1997;10:107-12.

4 Lee T-S, Hsu W-C, Chen C-J, et al. Etiologic study of young ischemic stroke in Taiwan. Stroke 2002;33:1950-5.

5 Garbusinski JM, van der Sande MAB, Bartholome EJ, et al. Stroke presentation and outcome in developing countries. A prospective study in the Gambia. Stroke 2005;36:1388-93

6 Adams HP Jr, Kappelle $\sqcup$, Biller J, et al. Ischemic stroke in young adults. Experience in 329 patients enrolled in the lowa Registry of stroke in young adults. Arch Neurol 1995;52:491-5.

7 Kristensen B, Malm J, Carlberg B, et al. Epidemiology and etiology of ischemic stroke in young adults aged 18 to 44 years in Northern Sweden. Stroke 1997;28:1702-9.

8 Leys D, Bandu L, Henon $\mathrm{H}$, et al. Clinical outcome in 287 consecutive young adults (15 to 45 years) with ischemic stroke. Neurology 2002;59:26-33.

9 Nedeltchev K, der Maur TA, Georgiadis D, et al. Ischemic stroke in young adults: predictors of outcome and recurrence. J Neurol Neurosurg Psychiatry 2005;76:191-5.

10 Reddy KS. Cardiovascular diseases in non-western countries. N Engl J Med 2004;350:2438-40.
11 McKeigue PM, Ferrie JE, Pierpoint T, et al. Association of early-onset coronary heart disease in South Asian men with glucose intolerance and hyperinsulinemia. Circulation 1993;87:152-61.

12 Dhawan J, Bray CL, Warburton R, et al. Insulin resistance, high prevalence of diabetes, and cardiovascular risk in immigrants Asians. Genetic or environmental effect? Br Heart J 1994;72:413-21.

13 Hatano $\mathrm{S}$. Experience from a multi centre stroke register: a preliminary report. Bull WHO 1976:54:541-53.

14 Joseph A, Kutty VR, Soman CR. High risk for coronary heart disease in Thiruvananthapuram city: a study of serum lipids and other risk factors. Indian Heart J 2000;52:29-35.

15 Britton M, Carlsson A, de Faire U. Blood pressure course in patients with acute stroke and matched controls. Stroke 1986;17:861-4.

16 Lindsberg PJ, Roine RO. Hyperglycemia in acute stroke. Stroke 2004;35:363-4.

17 Kleinman Y, Korn-Lubetzki I. Serum lipids in acute stroke and a year later. Stroke 1997;28:1467.

18 Adams HP Jr, Bendixen BH, Kappelle $\sqcup$, et al. Classification of subtype of acute ischemic stroke. Definitions for use in a multicenter clinical trial. TOAST. Trial of Org 10172 in Acute Stroke Treatment. Stroke 1993;24:35-41.

19 Chobanian AV, Bakris GL, Black HR, et al. The Seventh Report of the Joint National Committee on Prevention, Detection, Evaluation, and Treatment of High Blood Pressure: The JNC 7 Report. JAMA 2003;289:2560-72.

20 Executive Summary of the Third Report of The National Cholesterol Education Program (NCEP) Expert Panel on Detection, Evaluation, and Treatment of High Blood Cholesterol In Adults (Adult Treatment Panel III). JAMA $2001 ; 285: 2486-97$

21 Alberti KG, Zimmet PZ. Definition, diagnosis and classification of diabetes mellitus and its complications. Part 1: diagnosis and classification of diabetes mellitus provisional report of a WHO consultation. Diabet Med 1998; 15:539-53.

22 International Diabetic Federation. IDF Worldwide definition of the metabolic syndrome. http://www.idf.org/home/index.cfm?node=1429 (accessed 29 June 2007).

23 Bharucha NE, Bharucha EP, Bharucha AE, et al. Case-control study of completed ischemic stroke in the Parsis of Bombay: a population-based study. Neurology 1988;38:490-2.

24 Sridharan R. Risk factors for ischemic stroke: a case control analysis. Neuroepidemiology 1992;11:24-30.

25 Wacholder S, Silverman DT, McLaughlin JK, et al. Selection of controls in casecontrol studies. II. Types of controls. Am J Epidemiol 1992;135:1029-41.

26 West DW, Schuman KL, Lyon JL, et al. Differences in risk estimations from a hospital and a population-based case-control study. Int J Epidemiol 1984;13:235-9.

27 Infante-Rivard C. Hospital or population controls for case-control studies of severe childhood diseases? Am J Epidemiol 2003;157:176-82.

28 Rohr J, Kittner S, Feeser B, et al. Traditional risk factors and ischemic stroke in young adults: the Baltimore-Washington Cooperative Young Stroke Study. Arch Neurol 1996:53:603-7.

29 You RX, McNeil JJ, O'Malley HM, et al. Risk factors for stroke due to cerebral infarction in young adults. Stroke 1997;28:1913-18.

30 Quzilbash N, Duffy SW, Warlow C, et al. Lipids and risk factors for ischaemic stroke-overview and review. Cerebrovasc Dis 1992;2:127-36.

31 Curb JD, Abbott RD, Rodriguez BL, et al. High density lipoprotein cholesterol and the risk of stroke in elderly men: the Honolulu Heart Program. Am J Epidemiol 2004; 160:150-7

32 Albucher JF, Ferrieres J, Ruidavets JB, et al. Serum lipids in young patients with ischaemic stroke: a case-control study. J Neurol Neurosurg Psychiatry 2000;69:29-33.

33 Lewis GF, Rader DJ. New insights into the regulation of HDL metabolism and reverse cholesterol transport. Circ Res 2005;96:1221-32.

34 Alagona C, Soro A, Westerbacka J, et al. Low HDL cholesterol concentration is associated with increased intima-media thickness independent of arterial stiffness in healthy subjects from families with low HDL cholesterol. Eur J Clin Invest 2003;33:457-63.

35 Koren-Morag N, Goldbourt U, Tanne D. Relation between the metabolic syndrome and ischemic stroke or transient ischemic attack: a prospective cohort study in patients with atherosclerotic cardiovascular disease. Stroke 2005;36:1366-71.

36 Milionis HJ, Rizos E, Goudevenos J, et al. Components of the metabolic syndrome and risk for first-ever acute ischemic nonembolic stroke in elderly subjects. Stroke 2005;36:1372-6.

37 Paek KW, Chun KH, Lee KW. Relation between metabolic syndrome and familial history of hypertension/stroke, diabetes, and cardiovascular disease. J Korean Med Sci 2006;21:701-8.

38 Vimaleswaran KS, Radha V, Mohan V. Thr54 allele carriers of the Ala54Thr variant of FABP2 gene have associations with metabolic syndrome and hypertrigyceridemia in urban South Indians. Metabolism 2006;55:1222-6.

39 Jha P, Chaloupka FJ. The economics of global tobacco control. BMJ 2000;321:358-61.

40 Asia Pacific Cohort Studies Collaboration. The effects of diabetes on the risks of major cardiovascular diseases and death in the Asia-Pacific region. Diabetes Care 2003;26:360-6. 